\title{
Vegetarianism and veganism in adolescence: Benefits and risks
}

\author{
Luiz Antonio Del Ciampo ${ }^{1 *}$ and Ieda Regina Lopes Del Ciampo ${ }^{2}$ \\ ${ }^{1}$ Department of Puericulture and Pediatrics, Faculty of Medicine of Ribeirão Preto of São Paulo University, São Paulo, Brazil \\ ${ }^{2}$ Medicine Department, Federal University of São Carlos, São Paulo, Brazil
}

\begin{abstract}
Vegetarianism and veganism are a broad terms that encompasses a diverse and heterogeneous range of dietary practices that avoid flesh foods. This food practice has been known for many centuries and, in last years, demand for vegetarian food have increased notably. Reasons for choosing a vegetarian diet include potential health benefits, economic status, religious and sociopolitical, ecological, ethical, environmental, etc, and many studies have shown health benefits associated with vegetarian diets. This article presents some characteristics related to the consumption of food of plant origin and its repercussion on the body and health of the adolescent.
\end{abstract}

\section{Introduction}

Vegetarianism is a broad term that encompasses a diverse and heterogeneous range of dietary practices that avoid flesh foods (meat, poultry, seafood) and their products, while vegetarian diet is defined as a "diet consisting wholly of vegetables, fruits, grains, nuts, and sometimes egg or dairy products" [1]. This food practice has been known for many centuries and, in last years, the number of consumers following a vegetarian diet and the demand for vegetarian food have increased notably in many countries [2].

According to the American Dietetic Association the vegetarian diet can be classified into three main types: 1) strict vegetarianism: excludes all kinds of animal food; 2) lactovegetarianism: in addition to vegetables includes milk and dairy products; 3 ) ovolactovegetarianism: in addition to vegetable includes milk, dairy products and eggs [3].

Reasons for choosing a vegetarian diet are varied and include potential health benefits, economic status, religious and sociopolitical, ecological, ethical, and environmental issues related to allocation of resources and animal rights $[4,5]$. The decision to use a vegetarian diet for health reasons might be strongly influenced by public perceptions of the health advantages, and clinical and epidemiological research studies have shown health benefits associated with vegetarian diets $[1,6]$. The types and composition of vegetarian diets also are varied and have important implications for the growth and development of adolescents.

Adolescence is widely recognized as the stage of development that occurs between childhood and adulthood and is characterized across cultures and species by the onset of puberty as well as unique neurobiological, social, and cognitive development [7]. It is a time to explore new roles and lifestyles, establish independence, and expres a unique identity. Interest in alternative lifestyles and diets, including vegetarianism, is a growing phenomenon in this age group. Furthermore, during adolescence, both males and females have a heightened sensitivity about their appearance and often experience pressure to conform to a cultural ideal, resulting in body dissatisfaction and experimentation with various weight loss methods [8]. Adolescents may adopt eating patterns, such as vegetarianism, as a way to establish an identity, expres values, and assert control over their lives $[9,10]$.
Nutritional needs during adolescence are influenced by the onset of puberty with associated increased growth rate and changes in body composition and organ systems. The recommended dietary energy requirements in adolescents are defined to maintain health, promote optimal growth and maturation, and support a desirable level of physical activity $[11,12]$.

Vegetarian diets can meet nutrient needs for growth and development when they are carefully planned with attention. In general vegetarian diets are rich in fibre, iron, folic acid, magnesium, vitamins C and E, n-6 polyunsaturated fatty acid, carotenoids, flavonoids, other phytochemicals and antioxidants. However, these diets are low in calories, total fat, n-3 PUFA, calcium, iodine, zinc, vitamins B12 and $\mathrm{D}[1,13]$.

While there is a range of health benefits associated with a vegetarian diet, there are potential risks as well. If vegetarian diets are not wellplanned, there may be an increased potential for specific nutrient deficiencies [2]. In addition to potential nutrient deficiencies, there is concern about the consistente pattern associating vegetarianism and disordered eating behaviors in adolescents [14].

With a good planning and careful attention, vegetarian can include in their diets all essential nutrientes [6]. In general, it has been observed that vegetarians had lower risks of obesity, hypertension, cardiovascular diseases, diabetes, arthritis, cancers (especially colon and prostate cancer) and fatal ischemic heart disease, thanks to protective substances found in fruits, vegetables, legumes, seaweed, seeds, whole grains, vegetable oils and other plant-based foods [15].

${ }^{*}$ Correspondence to: Luiz Antonio Del Ciampo, Department of Puericulture and Pediatrics, Faculty of Medicine of Ribeirão Preto of São Paulo University, São Paulo, Brazil, E-mail: delciamp@fmrp.usp.br

Key words: vegetarianism, veganism, adolescence, nutrition

Received: December 06, 2018; Accepted: December 28, 2018; Published: January 02, 2019 


\section{Energy}

Daily energy needs during adolescence vary according to sex and pubertal spurt, and can be easily obtained with any type of diet. Until the age of 14 years 2500 calories are sufficient for both sexes. However, boys over 15 years require 3000 calories while 2300 calories are sufficient for girls. It is important to note that a diet with a high fiber content may provide a sense of fullness before an adequate amount energy is ingested, and vegetarian diets that contain less than $15 \%$ of calories from fat can adversely impact on growth and development of children and adolescentes [16,17].

\section{Protein}

Protein is necessary for growth, imune function and tissue repair and its quality is determined by amino acid composition. The difference between proteins of plant and animal origin is the concentration of essential amino acids, and protein needs of vegetarians are met when the diet includes a variety of plant foods and caloric intake is adequate [18]. Animal foods are considered complete or high-quality proteins because they contain all nine essential amino acids while plant-based foods are usually deficient in one or more of the essential amino acids. The deficit of one plant food can be overcome by combining it with a complementary plant food that provides adequate amounts of the limited essential amino acid. Vegetarians should consume a wide variety of vegetables to obtain all the daily necessary amino acids [19]. The daily requirement for protein is $1.0 \mathrm{~g} / \mathrm{kg}$ for individuals aged 10 and 14 years, while adolescents older than 14 years require $0.8 / \mathrm{kg}$ [20]. The major plant food sources of protein are beans, lentils, cereals, nuts and seed, and each variety has diferent composition of essential amino acids and digestibility.

\section{Omega-3 fatty acids}

Fats should represent $35 \%$ of the daily caloric intake for the adolescent. Vegetarian diets generally are rich in omega- 6 fatty acids, but marginal in omega-3 fatty acids, unless the diet includes fish, eggs, or generous amounts of algae. Omega-3 fatty acids, which include eicosapentaenoic acid (EPA) and docosahexaenoic acid (DHA), or their precursor alpha-linolenic acid (ALA), are important for cardiovascular health and eye and brain development [11]. Vegetarians have lower blood levels of EPA and DHA than nonvegetarians [18].

Vegetarians can improve their n-3 nutritional status by regularly consuming good sources of ALA (flaxseeds, walnuts, chia seeds and their oils) and limiting intake of sources of linoleic acid (corn and sunflower oils). To enhance the conversion of ALA to EPA and DHA, vegetarians should be advised to ensure their diet contains sufficient proteins, pyridoxine, biotin, calcium, copper, magnesium, and zinc [18].

\section{Iron}

Up to 13 years of age adolescents need $8 \mathrm{mg} /$ day of iron, and this concentration increases to $12 \mathrm{mg}$ for boys and $15 \mathrm{mg}$ for girls age 14 to 18 years [20]. Potential consequences of iron deficiency include growth retardation, behavioral problems, impaired cognitive and motor development, and deficient immune response. Concerns about iron nutrition for vegetarian are related to the differences between heme and nonheme iron. Heme iron is more readily absorbed than nonheme iron and the absorption of nonheme iron is reduced greatly by some dietary components, whereas heme iron is little affected. Tannins and polyphenols present in coffee and tea form iron-tannate complexes that greatly reduce nonheme iron absorption while phytate of legumes, nuts, seeds, whole grains, and soy protein also bind with nonheme iron to form insoluble complexes and reduce its absorption [21,22].

Some strategies used to increase iron absorption include supplementation with ascorbic acid and avoidance of large intakes of tannin-containing teas. Thus the bioavailability of iron in a vegetarian diet can be enhanced by consuming ascorbic acid during a meal containing iron. Other organic acids in fruits and vegetables (citric, malic, lactic and tartaric acids), as well as carotenes andretinol, also enhance non-haem iron absorption [18]. Good plant sources of iron include legumes, dried fruits, soy, green leafy vegetables, bulgur, blackstrap molasses, and wheat germ. The widespread fortification of enriched breads, cereals, and pasta products has helped increase iron intake for adolescents.

\section{Zinc}

Zinc is found in foods of both animal and plant origin and its recommendations are $8 \mathrm{mg}$ /day for adolescent aged 10 to 13 years, while for adolescent females and males aged 14 to 18 years are $9 \mathrm{mg} /$ day and $11 \mathrm{mg} /$ day, respectively [20]. Good plant sources of zinc include whole grains, legumes, wheat germ, and nuts. Cereals are the primary source of zinc for adolescents that consume vegetarian diets without milk. Zinc deficiency can be associated with growth impairment and an increased risk of infections, particularly diarrhea and pneumonia $[23,24]$.

Sulphuramino acids, cysteine-containing peptides, hydroxy acids (present in fruits) and other organic acids present in fermented food may all increase zinc absorption. As with iron, procedures that activate the endogenous phytases present in cereals and pulses, like milling, sprouting, soaking, and sour-dough leavening, increase the bioavailability of zinc [18].

Phytates found in larger quantities in vegetarian diets bind zinc and reduce its bioavailability. Certain food preparation techniques, such as the soaking of sprouting beans, grains, and seeds, as well as leavening breads, can reduce binding of zinc by phytates and increase zinc bioavailability [25].

\section{Calcium}

Calcium is an essential mineral during growth when bone mass is expanding. Maintenance of adequate calcium intake is important throughout life to ensure peak bone mass accumulation, especially during periods of growth. Adolescent with stronger bones may experience fewer fractures and may be more resistant to the development of osteoporosis in later life [4]. The optimal daily dietary allowance of calcium for adolescents is $1300 \mathrm{mg}$ for those 10 to 18 years for skeletal growth; for losses in urine, sweat, and endogenous secretion; and to adjust for average calcium absorption efficiency [20]. Dairy products have been positively associated with bone health because of their calcium content. Low calcium intake leads to increased bone remodeling and increased risk of hip fracture. Milk is the most economical source of many limiting nutrients, especially calcium, potassium, and magnesium [12].

Several plant foods, particularly leafy vegetables pulses, nuts, kale, mustard greens, broccoli, bok choy, contain good quantities of calcium, however the bioavailability of this mineral is inversely proportional to the amounts of oxalate and phytate in the diet [18]. Calcium intake may need to be increased to overcome the effects of other dietary constituents that decrease calcium absorption (oxalate and phytate) or increase excretion (salt and protein). The diminished intake of animal 
protein by vegetarians may help their net calcium balance by decreasing urinary calcium loss. Animal protein, with sulfur-containing amino acids, produces an increased acid load and decreases renal tubular calcium reabsorption. Most of the calcium needs of adolescent that consume lactovegetarian or lacto-ovovegetarian diets can be met by low-fat milk and dairy products [26].

\section{Vitamin D:}

Occurs naturally in animal products such as liver, fatty ocean fish and egg yolks and may be lacking in vegetarian diets. Normal levels of vitamin D metabolites are necessary for adequate intestinal calcium, phosphate absorption, and bone formation. Vitamin D availability is a function of sunlight exposure and dietary intake and maintenance of normal serum vitamin D concentrations requires exposure to the sun on hands, arms, and face for 10 to 15 minutes/day for fair-skinned. In addition to sun exposure a dietary intake of 600 IU generally is recommended [20]. Vegetarians that do not consume milk are at risk for vitamin D. A dietary alternative is soy milk or breakfast cereal fortified with calcium and vitamin D. For those that can't get adequate sun exposure vitamin D supplementation needs to be considered [27].

\section{Vitamin B12}

Vitamin B12 is necessary for cell division, blood formation, and nervous system maintenance. Vitamin B12 deficiency can produce abnormal neurologic and psychiatric symptoms that include ataxia, psychoses, paresthesia, disorientation, dementia, mood and motor disturbances, and difficulty with concentration [28]. In addition individuals may experience too apathy and macrocytic anemia. Because vitamin B12 deficiency develops slowly, the vitamin status of vegetarians should be monitored regularly. Vitamin B12 (cobalamin) is only found in foods from animal sources (meat, fish, eggs, and dairy products) [28]. The RDA for cobalamin is $1.8 \mathrm{mcg}$ for 9 to 13 years and $2.4 \mathrm{mcg}$ for 14 to 18 years [20]. Sometimes, lacto-ovovegetarians and lactovegetarians can consume sufficient amounts of vitamin B12 from eggs, and milk products. Nonanimal sources of vitamin B12 include cereals, breads, nutritional yeast, and some fortified soy products. Because a high folic acid intake can hide the symptoms of B12 deficiency, neurologic symptoms may occur before detection [4]. Vegan and other vegetarians whose diet does not contain adequate vitamin B12 should consume a regular and reliable source of the vitamin, either in fortified foods or an oral B12 supplement. Commonly used B12-fortified foods include certain brands of nutritional yeasts, most ready-to-eat cereals, many meat analogs, and some milk alternatives. Vegetarian diets are usually high in folic acid intake, which could mask B12 deficiency anemia but still leave children at risk for neurological commitment [29].

\section{Fiber}

Vegetarians often have high dietary fiber intake and this can to promote normal laxation and possibly reduces the risk of developing certain diseases in adulthood. However, a diet with a great quantity of fiber can compromise dietary energy intake and reduce the bioavailability of minerals such as iron, calcium, and zinc due the presence of oxalates and phytates. A small loss of energy, primarily as fat, and protein may occur with a high intake of dietary fiber. Daily fecal energy loss is estimated to increase by 1 percent for every 6-gram increase in dietary fiber [30]. For adolescents aged 10 to 13 years the recommended fiber intake ranges from 26 to $31 \mathrm{~g} /$ day. This amount of dietary fiber should not have an adverse effect on mineral bioavailability, provided the dietary mineral intake is adequate. Intake of fiber that exceeds the recommendation is unlikely to decrease mineral bioavailability in vegetarian that consume a balanced diet from a variety of foods. Food processing, such as sprouting and fermentation, is known to degrade phytates, thereby enhancing the bioavailability of iron, zinc, and calcium [31].

\section{Conclusion}

According to the American Academy of Nutrition and Dietetics vegetarian diets, when well thought out, are appropriate for adolescentes [13]. A detailed dietary history, with specific attention to the variety of foods supplied (especially in cases of strict vegan or other restrictive diets), needs to be taken and appropriate advice given [25]. Besides that na appropriate education of the family and follow-up over time are essential.

A vegetarian diet could be used as a "socially acceptable" method to restrict intake and control weight has raised questions regarding the role of such a diet in the development or maintenance of disordered eating patterns or clinical eating disorders [26,32]. Because eating behaviors developed during adolescence can have health implications and influences on future chronic disease risk adolescents that follow a vegetarian diet as a means of weight management should be monitored closely for evidence of an eating disorder.

\section{References}

1. Cramer H, Kessler CS, Sundberg T, Leach MJ, Schuman D, et al. (201) Characteristics of Americans choosing vegetarian and vegan diets for health reasons. $J$ Nutr Educ Behav 49: 561-568. [Crossref]

2. Baroni L, Goggi S, Battaglino R, Berveglieri M, Fasan I, et al. (2019) Vegan nutrition for mothers and children: practical tools for healthcare providers. Nutrients 11: 5-37.

3. Messina VK, Burke KI (1997) Position of the American Dietetic Association: vegetarian diets. J Am Diet Assoc 97: 1317-1321. [Crossref]

4. Dunham L, Kollar LM (2006) Vegetarian eating for children and adolescents. J Pediatr Health Care 20: 27-34. [Crossref]

5. Barr SI (2015) Vegetarian diets. World Rev Nutr Diet 111: 53-57. [Crossref]

6. Petti A, Palmieri B, Vadalà M, Laurino C (2017) Vegetarianism and veganism: not only benefits but also gaps-A review. Progr Nutr 19: 229-242.

7. Orukibich J, Nassaw K, Mori K (2005) Comments on defining adolescence. Psychol Rep 97: 737-738. [Crossref]

8. Larsen B, Luna B (2018) Adolescence as a neurobiological critical period for the development of higher-order cognition. Neurosci Biobehav Rev 94: 179-195.

9. Dahl RE (2004) Adolescent brain development: a period of vulnerabilities and opportunities. Keynote address. Ann N Y Acad Sci 1021: 1-22. [Crossref]

10. Robinson-O'brien R, Perry CL, Wal MM, Story M, Neumark-Sztainer N (2009) Adolescent and young adult vegetarianism: better dietary intake and weight outcomes but increased risk of disordered eating behaviors. J Am Diet Assoc 109: 6486-6455.

11. Craig WJ (2009) Health effects of vegan diets. Am J Clin Nutr 89: 1627S-1633S [Crossref]

12. Weaver CM (2009) Should dairy be recommended as part of a healthy vegetarian diet? Point. Am J Clin Nutr 89: 1634S-1637S. [Crossref]

13. Melina V, Craig W, Levin S (2016) Position of the Academy of Nutrition and Dietetics: Vegetarian Diets. J Acad Nutr Diet 116: 1970-1980. [Crossref]

14. Marsh K, Zeuschner C, Saunders A (2012) Health implications of a vegetarian diet: A review. Am J Life Med 6: 250-267.

15. Strohle A, Waldmann A, Wolters M (2006) Vegetarian nutrition: Preventive potential and possible risks: Part 1: Plant foods. Wien Klin Wochenschr 118: 580-593. [Crossref]

16. Panebianco SM (2007) The merits and pitfalls of vegetarianism. Explore (NY) 3: 55-58. [Crossref]

17. Appleby PN, Key TJ (2016) The long-term health of vegetarians and vegans. Proc Nutr Soc 75: 287-293. [Crossref] 
18. Agnoli C, Baroni L, Bertini I, Ciappellano S, Fabbri A, et al. (2017) Position paper on vegetarian diets from the working group of the Italian society of human nutrition. Nutr Metab Cardiovasc Dis 27: 1037-1052. [Crossref]

19. Messina V, Mangels AR (2001) Considerations in planning vegan diets: children. $J \mathrm{Am}$ Diet Assoc 101: 661-669. [Crossref]

20. Institute of medicine (2011) Dietary reference intakes. National academic press, Washington.

21. Collings R, Harvey LJ, Hooper L, Hurst R, Brown TJ, et al. (2013) The absorption of iron from whole diets: a systematic review. Am J Clin Nutr 98: 65-81. [Crossref]

22. Śliwińska A, Luty J, Aleksandrowicz-Wrona E, Małgorzewicz S (2018) Iron status and dietary iron intake in vegetarians. Adv Clin Exp Med 27: 1383-1389. [Crossref]

23. Foster M, Samman S (2015) Vegetarian diets across the lifecycle: impact on zinc intake and status. Adv Food Nutr Res 74: 93-131. [Crossref]

24. Lönnerdal B (2000) Dietary factors influencing zinc absorption. J Nutr 130: 1378S-83S [Crossref]

25. Amit M (2010) Vegetarian diets in children and adolescents. Paediatr Child Health 15 : 303-314. [Crossref]
26. Chiplonkar SA, Tupe R (2010) Development of a diet quality index with special reference to micronutrient adequacy for adolescent girls consuming a lacto-vegetarian diet. J Am Diet Assoc 110: 926-931. [Crossref]

27. Grant JD (2017) Time for change: Benefits of a plant-based diet. Can Fam Physician 63: 744-746. [Crossref]

28. Wang H, Li L, Qin LL, Aong Y, Vidal-Alaball J, et al. (2018) Oral vitamin B12 versus intramuscular vitamin B12 for vitamin B12 deficiency. Cochrane Database 3 CD004655. [Crossref]

29. Rizzo G, Laganà AS, Rapisarda AM (2016) Vitamin B12 among vegetarians: Status, assessment and supplementation. Nutrients 8: 767-790. [Crossref]

30. Dinu M, Abbate R, Gensini GF, Casini A, Sofi F (2017) Vegetarian, vegan diets and multiple health outcomes: a systematic review with meta-analysis of observational studies. Crit Rev Food Sci Nutr 57: 3640-3649. [Crossref]

31. Schürmann S, Kersting M, Alexy U (2017) Vegetarian diets in children: a systematic review. Eur J Nutr 56: 1797-1817. [Crossref]

32. Timko CA, Hormes JM, Chubski J (2012) Will the real vegetarian please stand up? An investigation of dietary restraint and eating disorder symptoms in vegetarians versus non-vegetarians. Appetite 58: 982-990. [Crossref]

Copyright: (C2019 Del Ciampo LA. This is an open-access article distributed under the terms of the Creative Commons Attribution License, which permits unrestricted use, distribution, and reproduction in any medium, provided the original author and source are credited. 